http://dx.doi.org/10.21611/qirt.1992.010

\title{
Computer-based thermographic system
}

\author{
by B. WIECEK(*), M. GRECKI(*) and J. PACHOLIK(*)
}

(") Politechnika Lodzka, Instytut Elektroniki, ul. Stefanowskiego 18/22, 90-924 LODZ, Poland

\begin{abstract}
Computer-based thermography system developed lately, is presented. It allows to record and process thermal images captured by electrically-cooled Hughes-40TE thermal camera. The system can be conllgured as a stand-alone portable unit, equipped with printers, different kinds of displays, disks, etc. In a laboratory version, it is a powerful, PC486-compatible machine with a special computer-card interface, which gives enormous possibilities of thermal image processing and measurement automation. The \$yglem is adapted to widely used AGEMA-compatible thermography equipment as well.
\end{abstract}

\section{Introduction}

In many practical cases the possibilities of image processing offered by standard thormographic systems are not sufficient. In a majority of thermography applications, the dodlcated computer support is required. Typical thermovision camera is equipped with portable computer which records and converts thermal images into the form very convenient for uger interpretation. Processor calculates the temperature from the spectral power density of the incoming thermal radiation, compensates the drift errors caused by ambient temperature ohanges, displays images, etc. These basic camera functions are very useful in standard Industrial thermal investigations, but not sufficient for more advanced measurements, aspecially in semiconductor design domain. First of all, high computational power is demanded as well as high-capacity system memory for captured images.

The varying emissivity of the viewed object causes significant temperature measurement incorrectness. According to certain and finite camera scanning rate, the time delay of a few tens of milliseconds between the first and the last picture element in the thermal image, is not negligible. In effect, it means that temperature distribution displayed on the screen is caused not only by varying-in-time thermal process itself, but by infrared sensor shipment as well. Practically, we can effectively trace thermal processes, if the temperature changes during the scanning time are lower than the camera sensitivity. Additionally, computer-controlled thormographic system gives a possibility for advanced image processing, e.g. noise cancelling, flltoring, intensifying, etc. [2]. Powerful computer support makes the thermovision very versatile aven in areas, where its use has not been considered yet.

A dedicated software is developed to support either static or transient states surveys, partlcularly for semiconductor power devices [2].

\section{System overview}

Computer-controlled thermography system consists of the camera, processing unit and PCcompatible computer as shown in figure 1. The computer is equipped with special interface designed in form of computer-card (figure 2). The interface allows to capture the thermal irnages and store them in the system memory. In Hughes-40TE electrically cooled Thermal Video System, the digital data corresponding into the radiation intensity, generated by six camera detectors, is transferred to the computer in two phases. In the first one the data are tored in the intermediate, cache memory. In order to fasten its transfer, during the next phase, data is being sent to the computer memory in the DMA channel. In this approach, large memory buffer is not required. 
For Hughes-40TE cameras, an inactive part of scanning line where no image is generated) is long and takes approximately $50 \%$ of total scanning time, which allows to reactivate the interface elements (DMA counters, hand-shaking circuits, etc.) as well as to perform some calculation by the processor. The maximum recording rate depends upon image scanning time in the camera, and can not exceed 20 images/s. It denotes the practical limit of slow-varying thermal processes surveys with the use of thermography, however for rapid dynamic investigations, new methods of scanning time shortening have been developed lately [3].

The system can record images in so-called real-time mode with the maximum rate. In this case all images are transferred into the computer memory. Every sample requires 8196 byte of memory, and so, the number of stored images is limited by the size of system memory. Typically, we can easily record up to 400 images. This gives approximately 20 s tracking time of the thermal process. On the other hand, for slower processes, the images are sent and stored directly in the mass memory devices, i.e. floppy or hard disk. This allows to record much more image samples and substantially lengthen the tracking time.

In this case, the minimum repetition time of stored images depends upon the transfer rate of the disk. Additionally, the image compression algorithms can reduce size of the data, and in result shorten the time of storing the images. Recording can be initialized by external electric signal or given temperature level, what is often being required in industrial applications.

In many cases, output signals generated by infrared sensors are very noisy, especially when camera is used to measure low-temperature objects. This effect is more noticeable in electrically cooling systems, where the temperature of the detector is not so low. Noise reduction algorithms are implemented in the system mentioned here. The easiest method consists in averaging of the sequence of the images, but unfortunately, this time-consuming procedure is only recommended to static-state surveys. The more advanced method uses the two-dimensional digital filters [2]. Some examples of image processing are shown in figure $A^{*}$. Non-linear median as well as sigma filtering are implemented in the system.

In order to use the disk space efficiently, especially in the case of recording the transient processes, the data loseless image compression algorithms have been worked out. The size reduction factor depends upon the image complexity, but usually it is around 3 . On the other hand, the computation time of the image compression algorithms has to be considered, because of its significant contribution in total recording time.

The presented system ensures displaying either the single thermal image or their consecutive sequence in form of animated film, one-by-one. Obviously, displaying rate depends upon the computer speed. The good results of flashing the images on the screen has been obtained with the use of a typical PC-AT/12MHz. A cursor moving on the screen allows to read out the temperature in every image point. The temperature values can be given in Celsius, Kelvin and Fahrenheit scale. The horizontal and vertical intersections provide another way of fast thermal image interpretation. The middle region of the image can be zoomed by factor 2 . Three-dimensional color diagrams give the next outlook of temperature distribution. Additionally, the isotherms are available. They are very useful for a system equipped with monochrome screen and printer.

The system can be configured either as a laboratory or portable unit. The laboratory option is based on powerful PC486 computer equipped with color monitor and printer. The portable system in constructed from PC/XT sub-assemblies. It is equipped with two floppy disks (1.2MB/360KB), built-in keyboard, alphanumeric display, monochrome screen, printer, and mouse. A interactive software written in C language, works in MS-DOS environment. This ensures full transferability of thermal investigation results in PC computer family.

\section{Technical Specifications}

Minimum repetition time: - laboratory unit 
- portable unit

$50 \mathrm{~ms}$ - real-time mode

$1.2 \mathrm{~s}$

Maximum number of the recorded images:

- laboratory unit

- portable unit

Image resolution

Triggering

practically unlimited

200 images $/ 2$ disks

$1.2 \mathrm{MB}$ and $360 \mathrm{kB}$

512 pixels *240 lines * 16 colors

keyboard-controlled,

external signal,

temperature level

Display

- laboratory unit

- portable unit

EGA, VGA (color)

Hercules (isotherms)

\section{Conclusions}

Thermography is a contactless method of thermal inspection especially recommended to the inaccessible objects. The element being tested can work permanently, in its normal conditions. The only visual contact between camera and investigated device is required. Sometimes, it leads to case removal, that may involve heat sinks changing, and in consequence different powor dissipation. With the help of powerful computer support automatic emissivity equalisation 6an easily be implemented.

The system presented in this work can record thermal images in real-time mode at the maximum rate of 20 images/s. Data compression algorithms substantially speed up recording and increase system capacity. The use of these methods is especially recommended to real lime recording, where large memory and disk spaces are required. In practice, with the help of image compression algorithm w can easily reduce memory requirement to $30 \%-50 \%$.

Two-dimensional signal processing algorithms are implemented particularly, for noise eancelling in industrial environment applications.

\section{REFERENCES}

(1) Tho Probye Series 4000 Thermal Video System, Hughes Aircraft Co., User Operating Manual.

[I] Lucinski (J.), Napieraiski (A.), Wiecek (B.), Grecki (M)., Pacholik (J.). -Methods, Algorithms and Elements of Thermography System for Surveys and Design Power Devices and Circuits, etoooclally for Transient States. Part 1 and 2, Technical University of Lodz-Poland, 1990-1991, wök supported by Polish Ministry of National Education.

[1] Wiocek (B.), Grecki (M). -Two-Dimensional Modelling of P-N-P-N Power devices in ONWtate and its Thermography Verification. IASTED Conf.Proc. Modelling, Identification, Control, Innebruck, Feb. 1991.

(4) Wlecek (B.) - The Methods and Circuits for Thermal Image Conversion, Patents: P-287928, P.276525, Technical University of Lodz, 1989,1990. 
http://dx.doi.org/10.21611/qirt.1992.010

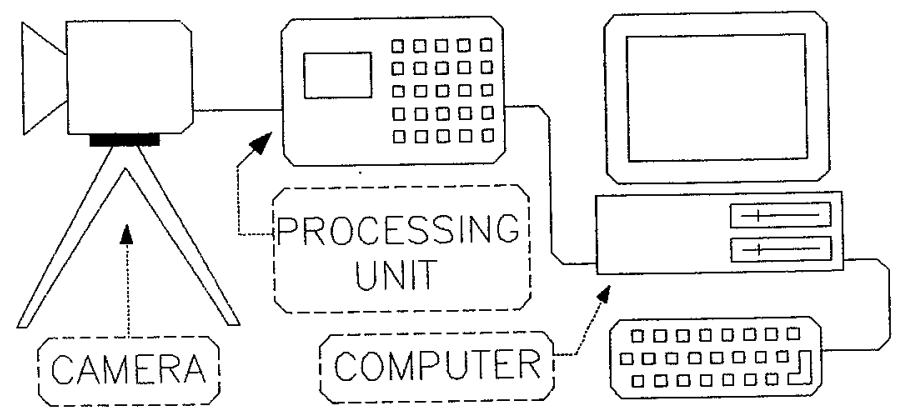

Fig.1. - IRS 2000 computer-controlled thermography system

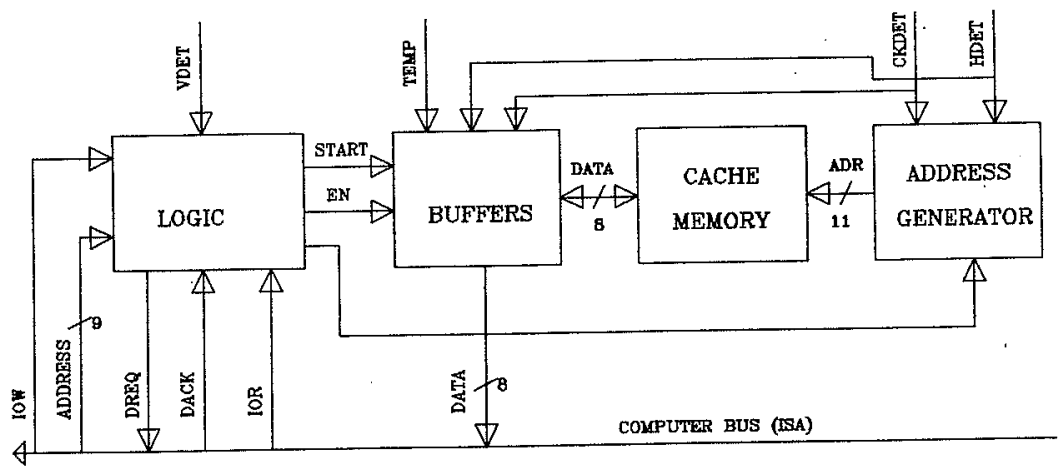

Fig. -2. Interface block diagram 